\title{
Possible ameliorative effect of colchicine on the prevention of cytokine storm and its associated hyper-inflammation in patients with COVID-19
}

 \\ ${ }^{1}$ Thalassemia and Hemoglobinopathy Research Center, Research Institute of Health, Ahvaz Jundishapur University of \\ Medical Sciences, Ahvaz, Iran \\ ${ }^{2}$ Department of Internal Medicine, Ahvaz Jundishapur University of Medical Sciences, Ahvaz, Iran \\ ${ }^{3}$ Medicinal Plant Research Center, Ahvaz Jundishapur University of Medical Sciences, Ahvaz, Iran
}

\section{Correspondence to:}

Mohammad Bahadoram,

Email: mohammadbahadoram@

yahoo.com

Received: 17 April 2020

Accepted: 1 May 2020

ePublished: 4 May 2020

\section{Citation: Keikhaei B,} Bahadoram M, Rajaei E, Alikhani K, Helalinasab A. Possible ameliorative effect of colchicine on the prevention of cytokine storm and its associated hyperinflammation in patients with COVID-19. J Prev Epidemiol. 2020;5(1):e02. DOI:10.34172/jpe.2020.02

\section{Core tip}

Colchicine, due to its anti-inflammatory and anti-fibrotic effects, may be suitable for prevention of cytokine storm and fibrosis in COVID-19

Keywords: Colchicine, Cytokine storm, Inflammation, COVID-19

A s of March $12^{\text {th }}, 2020$, coronavirus 2019 (COVID-19) has been confirmed in 12, 5045 persons worldwide, with a mortality rate of about $3.7 \%$ (1). Therefore, there is an urgent need for effective treatment. The current focus has been on developing new drugs, including antivirals and vaccines.

The current outbreak of lower respiratory tract infections, including respiratory distress syndrome, has been detected the third outbreak in the last two decades of an animal-to-human coronavirus leading to such a major epidemic.

Based on phylogenetic and taxonomic research, this virus has been officially classified as the sister of coronavirus acute respiratory syndrome (SARS-CoVs) identified as Acute Respiratory Syndrome Corona-2 (SARS-CoV-2) (2).

Current COVID-19 management is supportive care while the leading causes of death are respiratory failure due to acute respiratory distress syndrome (ARDS) and multiple organ failure (3). Evidence suggested that a subset of patients with severe COVID-19 may have cytokine storm syndrome too.

Hemophagocytic lymphocytosis, often referred to as "cytokine storm", is characterized by very severe and fatal hypercytokinemia with multiple organ failure (4). Mehta et al noted that the cytokine profile is similar to that seen in hemophagocytic lymphocytosis in patients with the acute phase of COVID-19 (5).

Hemophagocytosis is a process seen in severe inflammatory diseases such as sepsis, systemic inflammatory response syndrome, and multi-organ dysfunction syndrome, influenza, malaria, and leishmaniasis. In this phenomenon serum levels of cytokines and chemokines such as IFN $\gamma$, IL1 $\beta$, IL6, IL12, IL18, tumor necrosis factor- $\alpha$ (TNFa) are going to rise. These cytokines stimulate and activate immune cells, produce more cytokines, and eventually cause cytokine storm (5).

According to the study by Huang et al, in the SARS-CoV cytokine storm, the level of IFN $\gamma$ and related cytokines rose significantly. Also, the cytokine level had a positive and direct relationship with the neutrophil recruitment to the lung and aggravate the inflammation (6). Therefore, despite the benefits of neutrophils for diminishing infection and pathogens, over-calling them, will lead to an uncontrolled release of reactive oxygen species, bactericidal proteins, and their own cytokines and chemokines which will lead to other immune cells recall with a high-inflammatory reaction (7).

Evaluation of mortality in a multicenter retrospective study of 150 patients with 
COVID-19 in Wuhan, China, showed high levels of ferritin (mean $1297.6 \mathrm{ng} / \mathrm{mL}$ versus mean $614 \mathrm{ng} / \mathrm{mL}$ in survivors) and IL-6, indicating that mortality is likely to be due to increased inflammation caused by the virus (8).

Therefore, it is recommended to use available treatment with a proven immunologic profile for treating excessive inflammatory response and reduce mortality.

In China, during a controlled multicenter trial of tocilizumab administration as an IL-6 receptor blocker, licensed for cytokine storm syndrome, its beneficial effects for patients with COVID-19 pneumonia with increased levels of IL-6 was confirmed $(9,10)$.

As in patients with acute respiratory distress syndrome and the Middle East respiratory syndrome, corticosteroids are not usually recommended; while in patients with COVID-19 also corticosteroids may exacerbate lung injury (11). However, suppressing the immune system is likely to be helpful in a Hyper-inflammation status.

Another proposed supportive treatment is the possible ameliorative effect of colchicine as an inhibitor of leukocyte immigration in the prevention of cytokine storm and the resulting inflammation.

Colchicine is an alkaloid that was originally extracted from the bulb of the flowering plant called Colchicum Autumnale. Colchicine is an alkaloid used for Mediterranean family fever (FMF), gout, Behcet's disease, and recently, it has been used for other disorders such as cardiovascular diseases. However, poisoning caused by colchicine overdose is a life-threatening disease; therefore, it should be used with extreme caution in patients with renal or hepatic impairment. It is mainly excreted by the biliary system, intestines, and kidneys. Colchicine is metabolized by cytochrome 3A4. This cytochrome can be inhibited by various drugs that lead to colchicine poisoning during concomitant administration. Colchicine is effective in suppressing inflammation by various mechanisms. However, inhibition of neutrophil chemotaxis appears to be its main anti-inflammatory effect (12).

The primary effect of colchicine as an antimitotic drug is to destruct tubulin and inhibit mitotic cells in the metaphase stage of cell division. This leads to inhibition of inflammatory pathways and modulation of the immune system. Microtubules are involved in multiple cellular processes such as cytokine secretion, the activity of endothelial cells, immigration of leukocytes, and chemotaxis. Therefore, inhibition of tubulins leads to the inhibition of various anti-inflammatory pathways. The most important target for colchicine in regulating the immune system and reducing inflammation are neutrophils, endothelial cells, and macrophages, which act as a chain.

The most important anti-inflammatory mechanisms of colchicine are;

Inhibition of NALP3 inflammasome (NACHTLRRPYD-containing protein 3), and inhibition of pore production by inhibition of $\mathrm{P} 2 \mathrm{X} 7$ and $\mathrm{P} 2 \mathrm{X} 2$ pyrogenic receptors in macrophages, inhibition of neutrophil activation and their further recall by inhibiting the release of chemotactic factors such as IL-8, IL-1 and superoxide. Other mechanisms including, reduction the expression of E-selectin adhesion proteins on endothelial cells and L-selectin on neutrophils, thus reducing the interaction and adhesion of neutrophils with endothelial cells then lessening their immigration and invoking them more into the tissue. Colchicine also reduces the production of TNF- $\alpha$ by macrophages and their receptors on endothelial cells and inhibits endothelial proliferation by inhibiting the expression and production of vascular growth factor (13).

Kiraz and colleagues studied the effect of colchicine on inflammatory cytokines and selectins in FMF patients in a previous study. In this study, IL-6, IL-8, TNFa, and E and L-selectin levels were significantly reduced after two months of treatment with colchicine (14). Additionally, Paschke et al found that one of the mechanisms of colchicine in inhibiting the chemotaxis of neutrophils was to alter their elasticity by disturbing microtubules, thus modulating the neutrophil's passage and motility through cell membranes and thereby prevents excessive inflammation (15).

Therefore, colchicine has a restraining effect on several functions of leukocytes, such as adhesion, embolic motility, mobilization, and lysosome particle excretion. However, the greatest effect of colchicine is on the leukocyte chemotaxis. Among the mechanisms of colchicine effects, the inhibition of the chemotaxis occurred in the concentration as low as $1 \times 10-8 \mathrm{~mol} / \mathrm{L}$ of the drug. While higher doses are needed for other effects $(16,17)$.

\section{Conclusion}

Colchicine, due to its anti-inflammatory and anti-fibrotic effects, is expanding its therapeutic administrations. This study reviews the various anti-inflammatory patterns of colchicine and introduces it as one of the potential supportive therapies in the prevention of cytokine storm and its resulting hyper-inflammation, although to achieve greater certainty, modified designs and other trials are needed.

Conflict of interest

The authors declare that they do not have any conflict of interest.

Authors' contribution

All authors passed four criteria for authorship contribution based on recommendations of the International Committee of Medical Journal Editors.

Funding/Support

This study was financially supported by Ahvaz Jundishapur University of Medical Sciences, Ahvaz, Iran.

References

1. WHO. Coronavirus disease 2019 (COVID-19) situation report - 52. 2020. https://www.who.int/docs/default- 
source/coronaviruse/20200312-sitrep-52-covid-19. pdf?sfvrsn=e2bfc9c0_2

2. Gorbalenya AE. Severe acute respiratory syndrome-related coronavirus-the species and its viruses, a statement of the Coronavirus Study Group. BioRxiv. 2020

3. Huang C, Wang Y, Li X, Ren L, Zhao J, Hu Y, et al. Clinical features of patients infected with 2019 novel coronavirus in Wuhan, China. Lancet. 2020;395:497-506. doi: 10.1016/ S0140-6736(20)30183-5.

4. Ramos-Casals M, Brito-Zerón P, López-Guillermo A, Khamashta MA, Bosch X. Adult haemophagocytic syndrome. Lancet. 2014;383:1503-1516. doi: 10.1016/S0140-6736(13)61048-X.

5. Mehta P, McAuley DF, Brown M, Sanchez E, Tattersall RS, Manson JJ; HLH Across Speciality Collaboration, UK. COVID-19: consider cytokine storm syndromes and immunosuppression. Lancet. 2020;395:1033-1034. doi: 10.1016/S0140-6736(20)30628-0.

6. Huang KJ, Su IJ, Theron M, Wu YC, Lai SK, Liu CC, et al. An interferon-gamma-related cytokine storm in SARS patients. J Med Virol. 2005;75:185-94

7. Bordon J, Aliberti S, Fernandez-Botran R, Uriarte SM, Rane MJ, Duvvuri P, et al. Understanding the roles of cytokines and neutrophil activity and neutrophil apoptosis in the protective versus deleterious inflammatory response in pneumonia. Int J Infect Dis. 2013;17:e76-83. doi: 10.1016/j.ijid.2012.06.006.

8. Ruan Q, Yang K, Wang W, Jiang L, Song J. Clinical predictors of mortality due to COVID-19 based on an analysis of data of 150 patients from Wuhan, China. Intensive Care Med. 2020. doi: 10.1007/s00134-020-05991-x.

9. Chinese Clinical Trial Registry. A multicenter, randomized controlled trial for the efficacy and safety of tocilizumab in the treatment of new coronavirus pneumonia (COVID-19). 2020.

10. Richardson P, Griffin I, Tucker C, Smith D, Oechsle O, Phelan A, Stebbing J. Baricitinib as potential treatment for 2019-nCoV acute respiratory disease. Lancet. 2020;395:e30-e31. doi: 10.1016/S0140-6736(20)30304-4.

11. Russell CD, Millar JE, Baillie JK. Clinical evidence does not support corticosteroid treatment for 2019-nCoV lung injury. Lancet. 2020;395:473-475. doi: 10.1016/S01406736(20)30317-2.

12. Ben-Chetrit E. Colchicine. In: Hashkes P, Laxer R, Simon A eds. Textbook of Autoinflammation. Cham: Springer; 2019. p. 729-749.

13. Leung YY, Yao Hui LL, Kraus VB. Colchicine--Update on mechanisms of action and therapeutic uses. Semin Arthritis Rheum. 2015;45:341-50. doi: 10.1016/j. semarthrit.2015.06.013.

14. Kiraz S, Ertenli I, Arici M, Calgüneri M, Haznedaroglu I, Celik I, Pay S, Kirazli S. Effects of colchicine on inflammatory cytokines and selectins in familial Mediterranean fever. Clin Exp Rheumatol. 1998;16:721-4.

15. Paschke S, Weidner AF, Paust T, Marti O, Beil M, Ben-Chetrit E. Technical advance: Inhibition of neutrophil chemotaxis by colchicine is modulated through viscoelastic properties of subcellular compartments. J Leukoc Biol. 2013;94:1091-6. doi: 10.1189/jlb.1012510.

16. Phelps P. Appearance of chemotactic activity following intraarticular injection of monosodium urate crystals: effect of colchicine. J Lab Clin Med. 1970;76:622-31.

17. Caner JE. Colchicine inhibition of chemotaxis. Arthritis Rheum. 1965:8:757-64. doi: 10.1002/art.1780080438 